•综述・

\title{
航空航天遥感在物种多样性研究与保护中的应用
}

\author{
唐志尧 ${ }^{1,2 *}$ 蒋 炜 $^{2}$ 张 健 $^{3}$ 张新悦 $^{2}$ \\ 1 (北京大学生态中心, 北京 100871) \\ 2 (北京大学城市与环境学院, 北京 100871) \\ 3 (华东师范大学生态与环境科学学院, 上海 200241)
}

\begin{abstract}
摘要: 人类活动导致全球范围内生物多样性丧失日趋严重。物种多样性是研究最为深入以及最贴近生物多样性管 理的层次。物种多样性的研究往往受到多时空尺度生态过程的影响, 传统物种多样性调查方法受到人力物力影响, 局限性大, 物种多样性的研究与管理亟需整合不同来源的数据。遥感技术从传统的光学遥感阶段发展到不同平台、 不同维度相结合的多源遥感阶段, 并逐渐进入以高空间分辨率和高光谱为特征、以激光雷达为前沿发展方向的综 合遥感阶段。遥感技术因为其监测范围广、能监测人迹罕至地区以及长期可重复等特性, 为研究不同时空尺度的 生态学科学问题提供了更新更优的研究手段。本文围绕种群动态、种间关系与群落多样性、功能属性及功能多样 性以及生物多样性保护管理等生物多样性研究热点问题, 系统地论述了航空航天遥感技术在物种多样性研究与保 护领域的应用, 总结了航空航天遥感技术在研究与物种多样性有关的主要生态学问题中的机遇与挑战。我们认为 航空航天遥感技术利用多光谱甚至高光谱与激光技术从空中监测物种多样性, 从不同视角、基于不同光源提供了 物种多样性不同侧面的信息, 能够减小地面调查强度, 在大范围和边远地区的物种多样性调查研究中有着至关重 要的作用。依据光谱特性的物种判别以及依据激光雷达的三维结构量测将促进生物多样性的研究与管理, 加强遥 感学家和生物多样性研究者的沟通交流将有助于促进不同时空尺度的生物多样性与遥感技术的结合。
\end{abstract}

关键词: 遥感应用; 种群动态; 多样性; 功能属性; 保护

\section{Applications of satellite and air-borne remote sensing in biodiversity re- search and conservation}

\author{
Zhiyao Tang ${ }^{1,2^{*}}$, Minwei Jiang ${ }^{2}$, Jian Zhang ${ }^{3}$, Xinyue Zhang ${ }^{2}$ \\ 1 Institute of Ecology, Peking University, Beijing 100871 \\ 2 College of Urban and Environmental Sciences, Peking University, Beijing 100871 \\ 3 College of Ecology and Environmental Sciences, East China Normal University, Shanghai 200241
}

\begin{abstract}
Human activities has increasingly threatened the biodiversity of the world. Biodiversity science is a discipline that depends on scale, and research questions are often affected by the ecological process of multi-temporal scales. The traditional survey methods of biodiversity are often limited by human and material resources. It is therefore urgent to integrate different data sources in the biodiversity sciences. The remote sensing technique has developed from optical remote sensing to the multi-source remote sensing including different platforms combined with various sensors, and further to integrate the hyperspectral and hyper spatial resolution and light detection and ranging (LiDAR). The large coverage, the accessibility to remote areas, and the long-term repeatability of the remote sensing technique provide new and better solutions for studying ecological and scientific issues at different temporal and spatial scales. In this paper, we review the opportunity and challenges in the application of remote sensing in biodiversity sciences and conservation practices. Specifically, we focus on the applications of remote sensing in the issues related to the population dynamics, species interaction and community diversity, functional traits and functional diversity and biodiversity management. We suggest that the satellite and airborne remotes that employed multi-band or hyperspectral, high spatial resolution and LiDAR provide biodiversity information from different scopes, and
\end{abstract}

收稿日期: 2018-07-19; 接受日期: 2018-08-28

基金项目: 国家重点研发计划重点专项(2017YFA0605101)

* 通讯作者 Author for correspondence. E-mail: zytang@pku.edu.cn 
will play essential roles in the investigation of biodiversity in large-scale and remote areas. In the near future, species discrimination technique based on spectral characteristics and structure detection based on LiDAR will improve our understanding of the biodiversity sciences and management. We suggest to strengthen the communication between remote-sensing scientists and biodiversity researchers to promote the application of remote sensing technologies in biodiversity research and at different temporal and spatial scales.

Key words: remote sensing application; population dynamics; biodiversity; functional trait diversity; conservation

全球范围内生物多样性丧失日趋严重(Brook et $\mathrm{al}, 2013)$ 。为应对这一问题, 准确地监测生物多样性 的现状及其变化格外重要。生物多样性包含遗传多 样性、物种多样性并且生态系统多样性三个层次, 其中物种多样性是研究最为深入以及最贴近生物 多样性管理的层次。传统生物多样性调查方法主要 基于地面调查, 受人力物力限制, 这一监测方法局 限于较小区域。遥感技术具有即时性、系统性、非 破坏性, 可长期标准化地重复对地观测, 同时因为 其覆盖范围广, 在大尺度监测和生物多样性要素评 价方面具有优势, 可补充大尺度生物多样性的估算 及其动态, 为认知生物多样性的形成与维持机制、 变化成因与结果, 制定科学可行的生物多样性保护 和管理机制, 并监督与评估保护措施的执行与成效 提供了可能, 为区域和全球生物多样性估计提供了 一种有效的解决方案(Turner et al, 2003)。

在全球尺度的生物多样性评估中, 遥感数据以 其能够全球覆盖和连续监测的优势备受关注 (Skidmore et al, 2015)。在全球生物多样性观测网络 (Group on Earth Observations Biodiversity Observation Network, GEO BON) 2012年提出的生物多样性 关键变量(essential biodiversity variables, EBVs)的度 量标准中就包括了多项与遥感相关的指标(Pereira et al, 2013; Pettorelli et al, 2016)。传统的生态遥感虽 然主要关注生态系统及更大尺度的生态现象(张扬 建等, 2017), 但依然有一些卫星遥感系统如美国陆 地探测卫星系统专题绘图仪(the Landsat Thematic Mapper, Landsat TM)、增强型专题绘图仪(the Enhanced Thematic Mapper Plus, ETM+)和地球观测系 统(Systeme Probatoire d'Observation de la Terre, SPOT)高分辨率多波段扫描仪等, 已经被用于各种 生态系统和不同生物类群的物种多样性监测(Kerr \& Ostrovsky, 2003)。近年来, 近地面遥感也开始被 用于生物多样性的监测, 以获得更高分辨率和更高
精度的估计(Turner, 2014; 郭庆华等, 2016)。随着高 分辨率和高光谱传感器、新型运载工具以及激光雷 达技术的不断涌现，遥感在实施跨越不同组织水平 的生物多样性监测任务方面的优势越来越明显 (Davies \& Asner, 2014; Kuenze et al, 2014; 郭庆华 等, 2016)。遥感平台也从航天遥感(卫星)、航空遥 感(飞机与无人机)逐渐扩展到车载、红外相机监测、 视频及声频监控等平台。

本文主要集中于航天与航空平台, 聚焦在物种 多样性层次, 总结遥感在物种多样性研究中的应用, 特别是种群分布与动态、群落物种组成及其相互作 用、植物功能属性与功能多样性以及多样性保护管 理等领域的应用及有关进展，以期促进不同时空尺 度的生物多样性研究与遥感技术的结合, 为大尺度 生物多样性研究与保护提供参考。同时由于物种多 样性保护也涉及到生态系统的管理, 本文还包含了 部分生态系统遥感研究的进展。

\section{种群分布与动态监测}

生物多样性监测是生物多样性科学的研究热 点问题之一(马克平, 2016)。遥感技术为监测大型动 植物的种群分布及其动态提供了平台, 扩展了生物 多样性监测的视角。航空照片是最早用于动植物种 群数量监测的遥感数据, 此后逐渐发展到多波段卫 星、高光谱影像以及多源遥感数据源。总体而言, 大 型动植物的种群分布监测遥感技术可以划分为直 接监测和间接监测两种方式。直接监测是基于遥感 手段, 直接获取动植物个体数量的监测形式; 间接 监测则通过对动植物栖息地、特定生境或痕迹的遥 感监测来估算种群数量。

\section{1 直接监测}

航空照片因其飞行高度相对较低, 获取的数据 空间分辨率较高, 可以直接从稀疏植被中辨识出大 中型动物如黑斑牛羚(Connochaetes taurinus)、非洲 
野牛(Syncerus caffer) 以及非洲象(Loxodonta africana), 从而常被用于种群数量与动态的监测 (Watson \& Turner, 1965), 这一方法长期应用于动物 监测。对于绝大多数物种而言, 即便是中高分辨率 的多波段光学遥感, 它们的空间分辨率都还太低, 直接监测种群大小依然存在诸多限制。但一些草食 动物会改变地物光谱, 这种光谱的差异可以被多波 段遥感例如陆地卫星系列(Landsat)的遥感影像探测 出来, 用于区分不同物种进而估算其种群数量 (Heitkonig et al, 2003)。有些动物虽然个体较小, 难 以直接探测, 但当它们聚集到一定规模时也能够被 卫星影像所探测。Schwaller等(2013)就利用了南极 地区阿德利企我(Pygoscelis adeliae)的集群特征, 在 Landsat影像上准确划分了它在南极地区的分布区。 Lynch和Schwaller (2014)发现在有充足的地面调查 资料支持下，利用Landsat影像甚至可以估算大尺度 的阿德利企鹅的数量。随着高分辨率遥感影像的快 速发展, 高分多波段卫星遥感逐渐取代航空摄影和 传统多波段遥感，并广泛用于不同类群的动物种群 数量的监测中。例如Fretwell等(2012)和McMahon等 (2014)利用快鸟卫星传感器(QuickBird)的全色波段 和多波段影像，结合地面观测数据，第一次估算了 全球帝企我(Aptenodytes fosteri)以及Macquarie岛象 海豹(Mirounga leonina)的数量。

\section{2 间接监测}

大部分动植物个体太小, 难以被遥感探头直接 探测, 但因为其中一些动物需要一些特定的生境或 者会留下足够尺寸的痕迹如巢穴, 这些生境或者痕 迹可以为遥感手段所探测, 成为早期动物种群遥感 监测的主要替代目标, 如Cheatheam (1977)通过航 片判读德克萨斯州黑尾犬鼠(Cynomys ludovicianus) 的巢穴, 估算其密度与分布。有些动物的巢穴在遗 弃后会发生植物群落演替, 探测处于不同演替阶段 的植被也被用于估算该物种的种群动态(Dalsted et al, 1981)。Loffler和Margules (1980)利用南澳毛吻袋 熊(Lasiorhinus latifrons)巢穴和周边土丘的反射光 谱与该地区的背景植被的反射光谱的差异, 在 Landsat的多波段影像上清查巢穴数量来估算澳大 利亚南部南澳毛吻袋熊的种群大小; 近期, 高分多 光谱影像如伊科诺斯(IKONOS)卫星影像被大量用 于动物巢穴监测(Sidle et al, 2012)。

针对那些难以直接通过遥感技术监测的小型
的动植物, 一些遥感指标如总初级生产力 (gross primary productivity, GPP)、净初级生产力(net primary productivity, NPP)、归一化植被指数(normalized difference vegetation index, NDVI)、数字高程、 冠层高度、土地覆盖类型、火干扰等成为了探究生 物多样性格局形成机制的重要数据源(Pau et al, 2012; Burley et al, 2016; Zellweger et al, 2016; Pausas \& Ribeiro, 2017)。结合物种-生境关系, 利用 遥感技术识别物种的潜在分布区是生物多样性监 测的重要途径。从20世纪80年代开始, 大量的研究 开始利用多波段遥感影像(特别是Landsat系列数据) 监测动物不同类群的潜在生境, 如哺乳类的大熊猫 (Ailuropoda melanoleuca)(De Wulf，1988)、鸟类 (Avery \& Haines-Young, 1990)甚至是昆虫(Tucker et al, 1985; Cowley, 2000; Kalluri et al, 2007)及珊瑚礁 (Mumby et al, 1997)等。此后，高光谱和高分遥感则 进一步推动了生物多样性的生境监测研究(GómezRodríguez et al, 2008; Sandino, 2017)。

多波段遥感手段的生境制图可以衍生出生境 异质性，从而被用于不同类群的动物多样性制图及 其群落结构的推算(Kerr et al, 2001)。快速发展的高 分和高光谱遥感的光谱异质性也成为快速评估物 种多样性的替代指标(Rocchini et al, 2010; Levanoni et al, 2011)。

快速发展的激光雷达技术可以探测地物的三 维结构, 将动植物生境监测从原有的土地覆盖类型 等特征扩展到植被结构特征, 为动物分布与行为研 究提供了全新视角(Davies \& Asner, 2014), 为研究 动物在不同植被结构下的行为以及对同一植被不 同层次的利用方式提供了绝佳手段，极大地促进了 动物行为生态学的研究(Goetz et al, 2007; Palminteri et al, 2012; Loarie et al, 2013; Davies et al, 2016a, b)。

\section{群落物种组成与物种间的相互作}

群落构建和物种共存机制是理解群落生物多 样性维持机制的核心，该研究离不开群落物种组成 以及群落内物种之间的相互关系调查(马克平, 2016)。传统的野外群落调查非常耗时耗力, 在一些 人迹罕至的边远地区甚至是不可能的。利用遥感影 像进行物种判别一直是生态遥感学家们的梦想 (McGraw et al, 1998)。高分、高光谱遥感、激光雷 达技术进行群落调查和物种判别, 为群落物种组成 
和多样性的研究节省了大量的人力和时间, 同时, 由于遥感数据提供了清晰的空间位置, 为种间关系 及邻体效应检测提供了便利。

\section{1 群落物种组成判别}

早在20世纪80年代初期, Myers和Benson (1981) 即尝试通过大比例尺彩色红外照片的纹理特征判 别热带雨林的物种, 但这些尝试都只能停留在局域 尺度上。基于不同物种的季相差异信息, Fagan等 (2015)在利用多时相的Landsat影像区分了人工林与 天然林中的不同树种。高光谱遥感可以检测到不同 物种光谱反射的微弱差异, 使得大范围内区分群落 中的物种组成成为可能。例如Clark等(2005)在实验 条件下, 利用高分和多源高光谱遥感判别孤立的多 种热带雨林乔木; 其后, Zhang等(2006)利用机载高 光谱数字影像(hyperspectral digital imagery collection experiment, HYDICE)区分了热带雨林中的树种, 并在地面植被样带调查的基础上, 类似影像被 Lucas和Carter (2008)用于美国密西西比州Horn岛的 维管束植物多样性制图。激光雷达技术提供的清晰 的植被垂直结构与分枝构型, 进一步推动了物种判 别研究。城市树木常常是以孤立木为主, 因此可以 利用机载激光雷达技术进行物种判别(Liu et al, 2017); Cho等(2012)结合高光谱、激光雷达数据以及 WorldView-2及QuickBird高分多波段影像，完成了 南非稀树草原中树木的分类; Hakkenberg等(2018) 将这一手段应用到更为复杂的温带森林, 利用机载 高光谱、激光雷达以及高分影像, 结合地面调查数 据, 完成了杜克森林维管植物组成及其多样性制图, 并进一步利用群落排序和聚类对群落进行划分, 研 究了群落中物种组成的周转(即ß-多样性)。

通过这些不同遥感技术的整合, Feret和Asner (2014)在研究秘鲁的亚马逊森林时发现, 具有高空 间分辨率的高光谱影像可以判别群落的物种组成、 样地的光谱变异与物种多样性, 不同样地之间的光 谱差异性与其ß-多样性也高度吻合, 他们据此提出 了光谱种 (spectral species) 分布以及光谱多样性 (spectral diversity)的概念。结合高分和高光谱遥感 以及激光雷达技术等多源遥感信息, 在较大范围内 区分丰富度较高的混交林的树种组成已经成为可 能, 以光谱种判别为基础的 $\alpha$-和 $\beta$-多样性计算方法 也已日趋成熟(Rocchini et al, 2018)。

\section{2 种间关系与邻体效应检测}

结合高分和高光谱遥感以及激光雷达技术的 多源遥感信息，可以获取空间定位清晰的群落物种 组成数据, 为物种的邻体关系研究提供了得天独厚 的条件, 并极大推动了生物多样性与生态系统功能 关系的研究。Xu等(2015)利用开放获取的高分 Google Earth数据, 发现干旱区植被斑块面积的频 率分布图比零模型所模拟结果要右偏(即面积大的 斑块更多), 他们认为这是因为干旱区的物种之间 存在互惠作用。Davies等(2016b)在激光雷达技术和 高光谱遥感的支持下，提取了南非克鲁格国家公园 (Kruger National Park)稀树草原中白蚁蚁冢以及15 种树木的空间位置，发现蚁冢减少了周边 $10 \mathrm{~m}$ 范围 内的树种密度, 但增加了适应低地生境的树种密度, 从而维持了稀树草原的树种多样性。虽然目前机载 激光雷达技术在森林中树木个体定位上还面临挑 战，但地面激光扫描系统已经可以测量群落内不同 个体的位置、大小、分支构型以及冠幅延展等特征, 不同时相的测量比较可以用于表征不同个体在群 落中的表现，这一技术在近期被广泛用于植物群落 中邻体竞争的研究。例如Seidel等(2015)尝试利用该 手段检验了邻体效应对水杉(Metasequoia glyptostroboides)人工林中树木径向生长的影响。Li等(2014a, b)将这一技术用于江西新岗山的中国亚热带森林生 态系统与服务功能(Forest Biodiversity and Ecosystem Functioning Experiment in Subtropical Forests in China, BEF-China)实验平台，发现这一方法适用 于对树高 > $40 \mathrm{~cm}$ 小苗的结构测量, 并发现该样地 中幼苗的生长已经受到了邻体竞争的影响。在欧洲 水青冈(Fagus sylvatica)成熟林中, Metz等(2013)和 Barbeito等(2017)利用这一技术分别研究了林内不 同物种的冠幅结构特征及邻体关系, 发现群落内各 树木种内竞争较大，而针叶树对欧洲水青冈的竞争 较弱; 混交林中的欧洲水青冈比纯林中具有更大的 冠幅，这种混交效应随着立地生产力的增加而升高; 他们还发现，欧洲水青冈对不同管理强度下的邻体 竞争压力具有很强的表型可塑性(Juchheim et al, 2017)。

\section{3 功能属性与功能多样性制图}

在群落尺度开展的功能生态学, 特别是其中的 物种功能属性与功能多样性, 是理解群落生物多样 
性维持机制的重要内容(马克平, 2016)。功能属性及 其多样性是联系生物多样性与生态系统功能的关 键因素, 是近年来生态学的研究热点之一(刘晓娟 和马克平, 2015)。然而, 由于野外测量功能属性工 作量很大, 目前大多数关于功能属性数据的收集局 限于局域尺度的物种层次。遥感技术的发展为获取 大范围的高质量的功能属性和功能多样性数据提 供了可能(Lausch, 2016)。

生活型是早期表征生物生活史策略的主要综 合指标，优势植物的生活型也是划分生态系统类型 的最直接指标, 是最早的功能属性指标。早期的植 物生活型的遥感判别主要是利用航空彩色红外照 片的色调和纹理特征 (Knipling, 1970; Jackson, 1986)。在多波段的数字影像中, 不同生活型植物, 特别是木本植物与草本植物之间、阔叶树与针叶树 之间、常绿树种与落叶树种之间, 近红外波段以及 红波段的反射率具有显著差异(这两个波段也广泛 用于构建各种植被指数), 为区分植物功能型提供 了基础。不同生活型在近红外和红波段的反射率差 异后来被人们利用美国国家海洋和大气管理局气 象卫星高分辨率扫描辐射探测仪(National Oceanic and Atmospheric Administration Advanced Very High Resolution Radiometer, NOAA-AVHRR)、中分 辨率成像光谱仪(Moderate Resolution Imaging Spectroradiometer, MODIS) 以及Landsat系列遥感影像 划分全球和区域尺度生物群区或植被类型的依据 (Ustin \& Gamon, 2010)。

植物的生活型是植物对环境适应的综合指标, 难以区分植物对具体环境胁迫的适应策略, 也难以 跟生态系统的功能直接联系。从20世纪90年代开始, 植物功能属性开始成为连接生物多样性与生态系 统功能的桥梁(Funk et al, 2017)。从20世纪80年代开 始, 生态学家们就开始尝试利用多光谱遥感来测量 植物叶片的化学属性(Curran et al, 1989)。高光谱遥 感和激光雷达技术的快速发展, 为大范围的功能属 性调查提供了技术基础。不同化学组分具有其相应 的光谱响应曲线，高光谱遥感便于人们检测不同的 化学组分, 从而可有效区分植被冠层叶片中的各种 化学组分的含量(Asner \& Martin, 2009)。激光雷达 可以穿透冠层, 对于测量群落的分层结构、分枝构 型、冠层高度与厚度、冠层密度、植株大小等植物 结构属性具有极大优势(Lim et al, 2003)。Asner等
(2015)结合高光谱和激光雷达技术，研究了安第斯 山 79 个样地的冠层叶片的 15 种化学成分含量以及 比叶重的空间分布; 并随后将该研究扩展到整个秘 鲁的热带雨林, 测量了 7 个功能属性(比叶重、叶氮 含量、叶磷含量、叶钻含量等), 获得了整个区域的 功能属性变异图以及功能多样性指数, 并基于这些 数据将研究区域划分为 36 个功能等级和 6 个功能组 (Asner et al, 2016, 2017)。Schneider等(2017)以瑞士 的温带森林为研究对象, 基于激光雷达和遥感数据 测量的森林植物形态和生理特征数据绘制了该区 域的功能多样性地图, 并验证了该方法的可行性。 在全球尺度上，星载激光雷达已经成为全球树高及 其限制因素研究的重要数据来源(Tao et al，2016; Zhang et al, 2016b)。多源遥感的结合已经可以测量 植物的大量的功能属性, 如叶绿素a, b、胡夢卜素、 氮、磷、水分、可溶性碳、酚类、木质素、纤维素、 碳、钙、硼铁、钾、镁等化学性状以及冠层高度、 比叶重、活(死)物质比、叶高度等结构性状(Asner et al, 2015; Jetz et al, 2016; Schneider et al, 2017)。

\section{物种多样性保护管理}

生物多样性保护的合理实施需要大量的实地 数据支持, 运用遥感可以对边远地区进行长期的重 复监测。连续大尺度遥感监测提供了生境退化以及 生境丧失的直接数据, 为快速评估物种分布区变化 或灭绝速率提供了便捷的手段。大量的遥感数据因 为开放获取的特性，已经成为生物多样性形成、维 持、保护与管理研究中的重要数据源, 被广泛应用 到外来种入侵管理、保护优先区判别以及保护有效 性评估等方面(Turner et al, 2015; Skidmore et al, 2015; Amano et al, 2018)。

\section{1 外来入侵种监测及其分布区预测}

外来物种入侵及生物多样性对气候变化的响 应是生物多样性科学研究的核心议题(马克平, 2016)。评估外来物种入侵现状及其潜在入侵区域, 是对外来入侵种控制和管理的基础。面对外来物种 入侵这一全球性的生态问题，研究者们利用多波段 遥感影像, 结合激光雷达技术和其他数据资料, 分 析反射光谱、群落结构, 对入侵种入侵过程和分布 进行监测和预测。

外来入侵种监测可以借助外来种与乡土种之 间的反射光谱、纹理特征以及物候特征的差异，其 
中, 反射光谱特征主要以单个像元为对象来判别入 侵种, 纹理特征主要依据一定窗口范围内一组像元 反射光谱的对比度来判别入侵种, 而物候特征则依 据相同像元在一定时间周期内的光谱变化来判别 入侵种(Bradley, 2014)。例如Everitt和Deloach(1990) 利用航空像片中像元的反射光谱和物候特征, 以及 $\mathrm{Ge}$ 等(2006)利用航空像片中像元的纹理特征, 分别 评估了北美不同地区的柽柳(Tamarix spp.)的入侵 状况, 都取得了较高的精度; 旱雀麦(Bromus tectorum) 是由欧亚大陆入侵到北美草原的一年生草本 植物, 比当地草本植物萌芽早, Peterson (2005)利用 两者的物候差异, 评估了该物种在美国内华达州的 入侵现状。下层入侵植物往往被冠层植物所遮盖, 难以直接监测, 需要通过其他途径进行监测。例如 金银木(Lonicera maackii)是从东亚入侵到北美落叶 阔叶林的灌木，一般比当地乡土灌木春季萌芽早而 秋季调落晚, Resasco等(2007)利用了春秋季节乔木 层落叶期间的Landsat TM和ETM+影像计算出植被 指数，估算了美国俄亥俄州的金银木的入侵面积。 这些方法还广泛应用到草地的灌木入侵研究中 (Stevens et al, 2017)。

虽然遥感不能直接判别昆虫及微生物的入侵 情况, 但一些微生物或入侵昆虫会毁坏植被冠层。 例如Townsend等(2004)利用Landsat影像评估了舞 毒蛾(Lymantria dispar)对美国东部栎林的损害, 发 现舞毒蛾的暴发与水体富营养化有密切关系; Olsson等(2012)整合了SPOT和MODIS数据, 通过对挪 威云杉(Picea abies)受害面积制图, 评估了烟煤菌 对瑞典南部挪威云杉的影响, 并新发现了一种名为 红皮云杉球蚧(Physokermes inopinatus)的入侵种。利 用多波段遥感对某些蚊虫生境的监测, 也可以用于 蚊虫的预防研究(Zou et al, 2006)。

传统多波段遥感光谱分辨率和空间分辨率都 较低, 高分多波段遥感使得入侵植物的判读更为直 接(Malahlela et al, 2015)。激光雷达技术本身很少直 接用于监测入侵物种, 但它所获取的群落结构特征 (群落垂直结构、生物量、叶面积指数)及其在不同 时期的变化为判别植物的入侵过程及其现状提供 了重要的支持(Levick et al, 2015; Balzotti \& Asner, 2017)。外来物种的光谱曲线一般与本地物种有所差 别, 高光谱遥感可以检测到不同物种光谱反射率的 微弱差异, 从而有助于准确地探测外来入侵种
(Underwood et al, 2003)。例如, 固氮植物一般具有 较高的叶片氮含量, Asner和Vitousek (2005)根据这 一特征, 利用高光谱遥感AVIRIS评估了夏威夷火 山国家公园中的入侵树种火杨梅(Myrica faya) 的入 侵现状; 一些入侵植物在开花或者结实期可能会表 现出不同的反射特征，也可用于入侵种监测。例如 Andrew和Ustin (2006)、Michelle和Glenn (2009)依据 白花和黄色花苞的高光谱曲线，分别检测到加州入 侵种宽叶独行菜(Lepidium latifolium)和爱达荷州入 侵种乳浆大戟(Euphorbia esula)。对于下层植物, Ghulam等(2014)将光学和微波遥感结合, 绘制了马 达加斯加岛两种亚冠层入侵植物的分布。 Tesfamichael等(2017)借助光谱仪, 利用多波段遥感 影像(Landsat 8, Sentinel 2A, SPOT 6, Pleiades 1B, WorldView-3)区分几种入侵种(Artemisia afra, Asparagus laricinus及Seriphium plumosum)及与之共生 的本土植物, 从而极大地扩大了多波段遥感在入侵 植物监测的应用范围。Skowronek等(2017)整合高光 谱遥感和地面调查资料，在物种分布模型的协助下 甚至可以监测苔藓植物的入侵。利用不同时期的植 被指数及其他相关指数, 则可以评估物种的入侵过 程(或病虫害的侵害过程)并预测入侵种的潜在入侵 区域(或病虫害的潜在损害区域)，从而有助于制定 预防方案(Jones et al, 2015): 例如Bradley和Mustard (2006)利用不同时期的Landsat MSS (the MultiSpectral Scanner)/TM/ ETM+影像, 研究了1973-2001年 间旱雀麦在内华达州的入侵过程及其潜在生境。

现有物种分布以及空间化的环境变量是预测 物种分布区的两大限制因素。遥感技术可以在两方 面都有所贡献，从而提高了物种分布模型的模拟精 度，能够用于预测外来入侵种的潜在分布，并为外 来入侵种的防治提供决策支持(Elith \& Leathwick, 2009)。不同尺度的遥感数据可以提供一系列实时 的、空间明晰的地表环境变量如土地覆盖、植被指 数、地表温度、土壤水分等, 是物种分布模型的重 要输入参数, 有效提高物种分布模型对物种潜在分 布的预测精度(He et al，2015)。例如Buermann等 (2008)利用MODIS影像和QuikSCAT反演的植被变 量(LAI、森林盖度等)预测南美洲安第斯山和亚马逊 的8种鸟类、哺乳动物和植物的空间分布, 发现通过 联合遥感变量、气候、地形等因子能够更精确地预 测这些物种的分布。Zimmermann等(2007)和Cord等 
(2014)采用不同的物种分布模型方法分别预测了不 同树种的分布格局, 也发现遥感数据与其他环境变 量的结合能够更好地预测物种的空间分布。除了这 些静态指标外, 遥感还可以提供动态的空间变量, 如利用不同季节的植被指数, 有助于判别物种的潜 在分布区(Cord \& Rodder, 2011)。

\section{2 保护优先区判别}

保护优先区是对某一地区，根据物种分布、面 临的威胁、濒危状况和生态系统保护优先性等指标 划分, 依据保护价值和保护优先程度设立的优先保 护地区(吴波等, 2006)。热点地区对生物多样性保护 有重要意义, 是优先重点保护的地区。如前所述, 遥感可直接监测某些物种的分布、动态, 以及某些 动物的行为, 并可以通过光谱异质性间接测量物种 及功能多样性, 特别是遥感可以针对人迹罕至的偏 远地区开展直接探测, 为这些地区的生物多样性监 测提供直接支撑(Pettorelli et al, 2016)。但这些方法 由于价格昂贵或者技术要求过高, 只能绘制小范围 的多样性分布图。一些开放获取的遥感资料依然通 过多样性制图研究生境制图, 从而可作为确立保护 优先区的重要代用资料。早期生物多样性热点地区 遥感判别的重要依据是物种丰富度与遥感实测指 标如归一化植被指数之间的关系(Fjeldsae et al, 1997), 其后则多利用Landsat制图探讨土地覆盖类 型或者生境异质性与多样性的关系, 通过这些关系 提取物种丰富度分布的热点地区, 进一步确定保护 优先区(Luoto et al, 2002)。对于一些特殊生境如湿 地森林, 遥感制图可以直接用于保护区的规划 (Wiens et al, 2009)。

\section{3 物种保护有效性评估}

为了保护全球的生物多样性, “爱知目标”(2020 年全球生物多样性目标)第11项将全球陆地生物多 样性保护的目标设立为截至2020年全球陆地保护 区覆盖率超过17\%。截至2016年全球保护区的覆盖 面积约为 $14.7 \%$ (Lewis et al, 2018)。在保护区覆盖率 这一目标即将达成的前提下, 现有保护区的保护有 效性对于实现生物多样性保护目标至关重要(Tang et al, 2011)。建立保护区的主要目标是减少人类活 动, 而土地覆盖变化是人类活动的最直接后果之 一。具有长时间序列的中高分辨率多波段遥感数据 在评估保护区阻止土地覆盖变化, 减少其他人类活 动的有效性方面具有显著的优势, 已经被广泛用于
森林保护区的评估。研究人员将遥感数据和地理信 息系统(Geographic Information System, GIS)相结合, 建立野生动物栖息地评估框架，将不同生境因子以 数据图层形式叠加分析, 对野生动物, 如斑羚 (Naemorhedus goral) (Hong et al, 2004)、大熊猫(肖 炏等, 2004)、黑猩猩(Pan troglodytes) (Tweheyo et al, 2004)等的生境保护进行了评估。在保护管理方面, 保护区巡视和资源监测是动植物自然保护区中必 不可少的工作内容。无人机遥感已经开始应用到森 林监测(Zhang et al, 2016a)和保护区的巡视中(Koh \& Wich, 2012), 红外相机也应用到野生动物跟踪监 测中(晏玉芗等, 2014)。

此外, 比较保护区内部不同时期的森林覆盖率 (Liu et al, 2001), 或者比较保护区及其周边地区毁 林速率的差异可以用于评估森林自然保护区的有 效性(Bruner et al, 2001; Adam et al, 2008; Joppa et al, 2008; Tang et al，2011)。在荒漠地区，辛利娟等 (2015)结合遥感影像数据和调查数据, 选择荒漠植 被盖度、涵养水源功能、珍稀濒危物种种类及物种 多样性等指标评估了安西荒漠类保护区保护成效, 构建了荒漠类自然保护区保护成效评估指标体系。 辛利娟等(2014)还利用遥感影像分析景观破碎化和 沙化程度, 结合样方监测数据分析物种多样性和初 级生产力, 评估了草地类型保护区的生态保护成 效。郑姚闽等(2012)利用湿地遥感制图数据和中国 湿地自然保护区保护价值评价数据库, 采用层次分 析法、四分位法等方法，对1978-2008年间的91个中 国国家级湿地自然保护区的保护成效进行评估，建 立了湿地自然保护区保护价值评价指标体系。

\section{结语}

遥感具有即时性、系统性、可重复性的特性，是 一种长期的、标准化的、空间清晰的对地观测手段, 同时因为覆盖范围广, 在大尺度监测和评价生物多 样性要素方面具有优势, 从而为估计区域和全球的 物种多样性提供了一种解决方案。高分遥感、高光 谱遥感以及机载设备的推广, 分别扩展了遥感影像 的空间分辨率、光谱分辨率以及时间分辨率, 而激 光雷达技术则进一步提供了地物的三维结构。这些 技术的结合, 极大地推动了遥感在生物多样性研究 与保护领域的应用，减小了地面调查的强度，特别 是在大范围以及边远地区的生物多样性清查过程 
中起到了不可替代的作用。但同时我们也需要注意 遥感在不同层次的生物多样性研究中尚处于辅助 地位, 多源遥感也还只是极具针对性地应用于一些 特定的对象和区域，生物多样性的遥感应用还面临 一系列的挑战。

(1)现有遥感技术能力有限, 无法精准识别物 种, 难以取代野外实地调查。虽然遥感技术可以极 大地提高野外调查的广度和深度, 并且利用高光谱 的遥感技术能够判别并监测一些物种的分布, 但是 因为物种识别能力有限, 现阶段几乎所有有关生物 多样性的遥感应用都还是在系统的野外调查的支 持下完成的。促进遥感技术与地面物种识别能力的 结合，探索物种判别的新方法势在必行。

(2)目前还无法依靠遥感手段进行基于群落物 种组成(flora)的低等级的植被类型划分。遥感技术 可以高效地探测地物的外貌和结构, 已经广泛应用 于探测植物群落的外貌, 并基于群落外貌 (physiognomy)对高等级的植被类型进行划分。遥感 技术的可重复性也使之成为研究植被动态的重要 手段。然而, 基于光谱的遥感手段仅能对冠层物种 进行识别且识别能力极其有限, 而激光雷达遥感主 要关注的是结构参数及外形, 无法对下层植物进行 有效监测。

(3)虽然遥感技术在许多方面为生物多样性研 究和保护提供了技术支持, 但遥感学家与生物多样 性研究者之间还存在较大的认识上的不匹配。例如 遥感学家对一些生物多样性概念术语的不熟悉, 可 能会造成他们对遥感在生物多样性监测过程中的 结果预期过于乐观，而生物多样性研究者可能并没 有意识到遥感技术在生物多样性监测过程中的应 用范围。因此, 亟需加强遥感学家与生物多样性研 究者之间的联系, 更有针对性地为生物多样性研究 设计遥感平台与传感器。

\section{参考文献}

Amano T, Székely T, Sandel B, Nagy S, Mundkur T, Langendoen T, Blanco D, Soykan CU, Sutherland WJ (2018) Successful conservation of global waterbird populations depends on effective governance. Nature, 553, 199-202.

Andam KS, Ferraro PJ, Pfaff A (2008) Measuring the effectiveness of protected area networks in reducing deforestation. Proceedings of the National Academy of Sciences, USA, 105, 16089-16094.

Andrew ME, Ustin SL (2006) Spectral and physiological uniqueness of perennial pepperweed (Lepidium latifolium). Weed Science, 54, 1051-1062.

Asner GP, Knapp DE, Anderson CB, Martin RE, Vaughn N (2016) Large-scale climatic and geophysical controls on the leaf economics spectrum. Proceedings of the National Academy of Sciences, USA, 113, E4043-4051.

Asner GP, Martin RE (2009) Airborne spectranomics: Mapping canopy chemical and taxonomic diversity in tropical forests. Frontiers in Ecology and the Environment, 7, 269-276.

Asner GP, Martin RE, Anderson CB, Knapp DE (2015) Quantifying forest canopy traits: Imaging spectroscopy versus field survey. Remote Sensing of Environment, 158, 15-27.

Asner GP, Martin RE, Knapp DE, Tupayachi R, Anderson CB, Sinca F, Vaughn NR, Llactayo W (2017) Airborne laser-guided imaging spectroscopy to map forest trait diversity and guide conservation. Science, 355, 385-389.

Asner GP, Vitousek PM (2005) Remote analysis of biological invasion and biogeochemical change. Proceedings of the National Academy of Sciences, USA, 102, 4383-4386.

Avery MI, Haines-Young RH (1990) Population estimates for the dunlin Calidris alpina derived from remotely sensed satellite imagery of the Flow Country of northern Scotland. Nature, 344, 860-862.

Balzotti CS, Asner GP (2017) Episodic canopy structural transformations and biological invasion in a Hawaiian forest. Frontiers in Plant Science, 8, 1256.

Barbeitoa I, Dassot M, Bayer D, Collet C, Drössler L, Löf M, Del Rio M, Ruiz-Peinado R, Forrester DI, Bravo-Oviedo A (2017) Terrestrial laser scanning reveals differences in crown structure of Fagus sylvatica in mixed vs. pure European forests. Forest Ecology and Management, 405, 381-390.

Bradley BA (2014) Remote detection of invasive plants: A review of spectral, textural and phenological approaches. Biological Invasions, 16, 1411-1425.

Bradley BA, Mustard JF (2006) Characterizing the landscape dynamics of an invasive plant and risk of invasion using remote sensing. Ecological Applications, 16, 1132-1147.

Brook BW, Ellis EC, Perring MP, Mackay AW, Blomqvist L (2013) Does the terrestrial biosphere have planetary tipping points? Trends in Ecology and Evolution, 28, 396-401.

Bruner AG, Gullison RE, Rice RE (2001) Effectiveness of parks in protecting tropical biodiversity. Science, 291, 125128.

Buermann W, Saatchi S, Smith TB, Zutta BR, Chaves JA, Milá B, Graham CH (2008) Predicting species distributions across the Amazonian and Andean regions using remote sensing data. Journal of Biogeography, 35, 1160-1176.

Burley HM, Mokany K, Ferrier S, Laffan FW, Williams KJ, Harwood TD (2016) Primary productivity is weakly related to floristic alpha and beta diversity across Australia. Global Ecology and Biogeography, 25, 1294-1307.

Cheatheam LK (1977) Density and distribution of the blacktailed prairie dog in Texas. The Texas Journal of Science, 29, 33-40. 
Cho MZ, Mathieu R, Asner GP, Naidoo L, van Aardt J, Ramoelo A, Debba P, Wessels K, Main R, Smit IPJ (2012) Mapping tree species composition in South African savannas using an integrated airborne spectral and LiDAR system. Remote Sensing of Environment, 125, 214-226.

Clark ML, Roberts DA, Clark DB (2005) Hyperspectral discrimination of tropical rain forest tree species at leaf to crown scales. Remote Sensing of Environment, 96, 375-398.

Cord A, Rodder D (2011) Inclusion of habitat availability in species distribution models through multi-temporal remotesensing data? Ecological Applications, 21, 3285-3298.

Cord AF, Klein D, Gernandt DS, de la Rosa JAP, Dech S (2014) Remote sensing data can improve predictions of species richness by stacked species distribution models: A case study for Mexican pines. Journal of Biogeography, 41, 736748.

Cowley MJR, Wilson RJ, Leon-Cortes JL, Gutierrez D, Bulman CR, Thomas CD (2000) Habitat-based statistical models for predicting the spatial distribution of butterflies and day-flying moths in a fragmented landscape. Journal of Applied Ecology, 37, 60-72.

Curran PJ (1989) Remote sensing of foliar chemistry. Remote Sensing of Environment, 30, 271-278.

Dalsted KJ, Sather-Blair S, Worcester BK, Klukas R (1981) Application of remote sensing to prairie dog management. Journal of Range Management, 34, 218-223.

Davies AB, Asner GP (2014) Advances in animal ecology from 3D-LiDAR ecosystem mapping. Trends in Ecology and Evolution, 29, 681-691.

Davies AB, Marneweck DG, Druce DJ, Asner GP (2016a) Den site selection, pack composition, and reproductive success in endangered African wild dogs. Behavioral Ecology, 27, 1869-1879.

Davies AB, Tambling CJ, Kerley GIH, Asner GP (2016b) Effects of vegetation structure on the location of lion kill sites in African thicket. PLoS ONE, 11, e0149098.

De Wulf RR, Goossens RE, McKinnon JR, Cai WS (1988) Remote sensing for wildlife management: Giant panda habitat mapping from Landsat MSS images. Geocarto International, 1, 41-50.

Elith J, Leathwick JR (2009) Species distribution models: Ecological explanation and prediction across space and time. Annual Review of Ecology, Evolution, and Systematics, 40, 677-697.

Everitt JH, DeLoach CJ (1990) Remote sensing of Chinese tamarisk (Tamarix chinensis) and associated vegetation. Weed Science, 38, 273-278.

Fagan ME, DeFries RS, Sesnie SE, Arroyo-Mora JP, Soto C, Singh A, Townsend PA, Chazdon RL (2015) Mapping species composition of forests and tree plantations in northeastern Costa Rica with an integration of hyperspectral and multitemporal Landsat imagery. Remote Sensing, 7, 56605696.
Feret JB, Asner GP (2014) Mapping tropical forest canopy diversity using high-fidelity imaging spectroscopy. Ecological Applications, 24, 1289-1296.

Fjeldsaê J, Ehrlich D, Lambin E, Prins E (1997) Are biodiversity "hotspots" correlated with current ecoclimatic stability? A pilot study using the NOAA-AVHRR remote sensing data. Biodiversity and Conservation, 6, 401-422.

Fretwell PT, LaRue MA, Morin P, Kooyman GL, Wienecke B, Ratcliffe N, Fox AJ, Fleming AH, Porter C, Trathan PN (2012) An emperor penguin population estimate: The first global, synoptic survey of a species from space. PLoS ONE, 7, e33751.

Funk JL, Larson JE, Ames GM, Butterfield BJ, Cavender-Bares J, Firn J, Laughlin DC, Sutton-Grier AE, Williams L, Wright J (2017) Revisiting the Holy Grail: Using plant functional traits to understand ecological processes. Biological Review, 92, 1156-1173.

Ge S, Carruthers R, Gong P, Herrera A (2006) Texture analysis for mapping Tamarix parviflora using aerial photographs along Cache Creek, California. Environmental Monitoring and Assessment, 114, 65-83.

Ghulam A, Porton I, Freeman K (2014) Detecting subcanopy invasive plant species in tropical rainforest by integrating optical and microwave (InSAR/PolInSAR) remote sensing data, and a decision tree algorithm. ISPRS Journal of Photogrammetry and Remote Sensing, 88, 174-192.

Goetz S, Steinberg D, Dubayah R, Blair B (2007) Laser remote sensing of canopy habitat heterogeneity as a predictor of bird species richness in an eastern temperate forest, USA. Remote Sensing of Environment, 108, 254-263.

Gómez-Rodríguez C, Bustamante J, Koponen S, Díaz-Paniagua C (2008) High-resolution remote-sensing data in amphibian studies: Identification of breeding sites and contribution to habitat models. Herpetological Journal, 18, 103-113.

Guo QH, Liu J, Li YM, Zhai QP, Wang YC, Wu FF, Hu TY, Wan HW, Liu HM, Shen WM (2016) A near-surface remote sensing platform for biodiversity monitoring: Perspectives and prospects. Biodiversity Science, 24, 1249-1266. (in Chinese with English abstract) [郭庆华, 刘瑾, 李玉美, 翟 秋萍, 王永财, 吴芳芳, 胡天宇, 万华伟, 刘慧明, 申文 明 (2016) 生物多样性近地面遥感监测: 应用现状与前 景展望. 生物多样性, 24, 1249-1266.]

Hakkenberg CR, Peet RK, Urban DL, Song C (2018) Modeling plant composition as community continua in a forest landscape with LiDAR and hyperspectral remote sensing. Ecological Applications, 28, 177-190.

He KS, Bradley BA, Cord AF, Rocchini D, Tuanmu MN, Schmidtlein S, Turner W, Wegmann M, Pettorelli N (2015) Will remote sensing shape the next generation of species distribution models? Remote Sensing in Ecology and Conservation, 1, 4-8.

Heitkonig IMA, Ringrose S, Epema GF, Bonyongo C, Veenendaal E, Stein A, Breeuwer A, van Hasselt M, Klop E, van Goethem J, Slot M (2003) Watching wildlife from 
space. In: International Wetlands Conference (eds Bernard T, Mosepele K, Ramberg L), Botswana.

Hong SK, Kim S, Cho KH, Kim JE, Kang S, Lee D (2004) Ecotope mapping for landscape ecological assessment of habitat and ecosystem. Ecological Research, 19, 131-139.

Jackson RD (1986) Remote sensing of biotic and abiotic plant stress. Annual Review of Phytopathology, 24, 265-287.

Jetz W, Cavender-Bares J, Pavlick R, Schimel D, Davis FW, Asner GP, Guralnick R, Kattge J, Latimer AM, Moorcroft P (2016) Monitoring plant functional diversity from space. Nature Plants, 2, 1-4.

Jones C, Song C, Moody A (2015) Where's woolly? An integrative use of remote sensing to improve predictions of the spatial distribution of an invasive forest pest the Hemlock Woolly Adelgid. Forest Ecology and Management, 358, 222-229.

Joppa LN, Loarie SR, Pimm SL (2008) On the protection of "protected areas". Proceedings of the National Academy of Sciences, USA, 105, 6673-6678.

Juchheim J, Annighofer P, Ammer C, Calders K, Raumonen P, Seidel D (2017) How management intensity and neighborhood composition affect the structure of beech (Fagus sylvatica L.) trees. Trees, 31, 1723-1735.

Kalluri S, Gilruth P, Rogers D, Szczur M (2007) Surveillance of arthropod vector-borne infectious diseases using remote sensing techniques: A review. PLoS Pathogens, 3, e116.

Kerr JT, Ostrovsky M (2003) From space to species: Ecological applications for remote sensing. Trends in Ecology and Evolution, 18, 299-305.

Kerr JT, Southwood TRE, Cihlar J (2001) Remotely sensed habitat diversity predicts butterfly species richness and community similarity in Canada. Proceedings of the National Academy of Sciences, USA, 98, 11365-11370.

Knipling EB (1970) Physical and physiological basis for the reflectance of visible and near-infrared radiation from vegetation. Remote Sensing of Environment, 1, 155-159.

Koh LP, Wich SA (2012) Dawn of drone ecology: Low-cost autonomous aerial vehicles for conservation. Tropical Conservation Science, 5, 121-132.

Kuenze C, Ottinger M, Wegmann M, Guo H, Wang C, Zhang J, Dech S, Wikelski M (2014) Earth observation satellite sensors for biodiversity monitoring: Potentials and bottlenecks. International Journal of Remote Sensing, 35, 65996647.

Lausch A, Bannehr L, Beckmann M, Boehm C, Feilhauer H, Hacker JM, Heurich M, Jung A, Klenke R, Neumann C (2016) Linking Earth Observation and taxonomic, structural and functional biodiversity: Local to ecosystem perspectives. Ecological Indicators, 70, 317-339.

Levanoni O, Levin N, Peer G, Turbe A, Kar S (2011) Can we predict butterfly diversity along an elevation gradient from space? Ecography, 34, 372-383.

Levick SR, Setterfield SA, Rossiter-Rachor NA, Hutley LB, McMaster D, Hacker JM (2015) Monitoring the distribution and dynamics of an invasive grass in tropical savanna using airborne LiDAR. Remote Sensing, 7, 5117-5132.

Lewis E, MacSharry B, Juffe-Bignoli D, Harris N, Burrows G, Kingston N, Burgess ND (2018) Dynamics in the global protected-area estate since 2004. Conservation Biology. https://doi.org/10.1111/cobi.13056 (accessed on 2017-11-23)

Li Y, Härdtle W, Bruilheide H, Nadrowski K, Scholten T, von Wehrden H, von Oheimb G (2014a) Site and neighborhood effects on growth of tree saplings in subtropical plantations (China). Forest Ecology and Management, 327, 118-127.

Li Y, Hess C, von Wehrden H, Härdtle W, von Oheimb G (2014b) Assessing tree dendrometrics in young regenerating plantations using terrestrial laser scanning. Annals of Forest Science, 71, 453-462.

Lim K, Treitz P, Wulder M, St-Onge B, Flood M (2003) LiDAR remote sensing of forest structure. Progress in Physical Geography, 27, 88-106.

Liu J, Linderman M, Ouyang Z, An L, Yang J, Zhang H (2001) Ecological degradation in protected areas: The case of Wolong Nature Reserve for giant pandas. Science, 292, 98101.

Liu L, Coops NC, Aven NW, Pang Y (2017) Mapping urban tree species using integrated airborne hyperspectral and LiDAR remote sensing data. Remote Sensing of Environment, 200, 170-182.

Liu XJ, Ma KP (2015) Plant functional traits-concepts, applications and future directions. Scientia Sinica Vitae, 45, 325-339. (in Chinese with English abstract) [刘晓娟, 马克 平 (2015) 植物功能性状研究进展. 中国科学: 生命科 学, 45, 325-339.]

Loarie SR, Tambling CG, Asner GP (2013) Lion hunting behaviour and vegetation structure in an African savanna. Animal Behavior, 85, 899-906.

Loffler E, Margules C (1980) Wombats detected from space. Remote Sensing of Environment, 9, 47-56.

Lucas KL, Carter GA (2008) The use of hyperspectral remote sensing to assess vascular plant species richness on Horn Island, Mississippi. Remote Sensing of Environment, 112, 3908-3915.

Luoto M, Kuussaari M, Toivonen T (2002) Modelling butterfly distribution based on remote sensing data. Journal of Biogeography, 29, 1027-1037.

Lynch HJ, Schwaller MR (2014) Mapping the abundance and distribution of Adelie penguins using Landsat-7: First steps towards an integrated multi-sensor pipeline for tracking populations at the continental scale. PLoS ONE, 9, e113301.

Ma KP (2016) Hot topics for biodiversity science. Biodiversity Science, 24, 1-2. (in Chinese) [马克平 (2016) 生物多样性 科学的热点问题. 生物多样性, 24, 1-2.]

Malahlela OE, Cho MA, Mutanga O (2015) Mapping the occurrence of Chromolaena odorata (L.) in subtropical forest gaps using environmental and remote sensing data. Biological Invasion, 17, 2027-2042.

McGraw JB, Warner TA, Key TL, Lamar WR (1998) High 
spatial resolution remote sensing of forest trees. Trends in Ecology and Evolution, 13, 300-301.

McMahon CR, Howe H, van den Hoff J, Alderman R, Brolsma $\mathrm{H}$, Hindell MA (2014) Satellites, the all-seeing eyes in the sky: Counting elephant seals from space. PLoS ONE, 9, e92613.

Metz J, Seidel D, Schall P, Scheffer D, Schulze ED, Ammer C (2013) Crown modeling by terrestrial laser scanning as an approach to assess the effect of aboveground intra- and interspecific competition on tree growth. Forest Ecology and Management, 310, 275-288.

Mitchell JJ, Glenn NF (2009) Subpixel abundance estimates in mixture-turned matched filtering classifications of leafy spurge (Euphorbia esuls L.). International Journal of Remote Sensing, 30, 6099-6119.

Mumby PJ, Green EP, Edwards AJ, Clark CD (1997) Coral reef habitat mapping: How much detail can remote sensing provide? Marine Biology, 130, 193-202.

Myers BJ, Benson ML (1981) Rainforest species on large-scale colour photos. Photogrammetric Engineering and Remote Sensing, 47, 505-513.

Olsson PO, Jönsson AM, Eklundh L (2012) A new invasive insect in Sweden Physokermes inopinatus: Tracing forest damage with satellite based remote sensing. Forest Ecology and Management, 285, 29-37.

Palminteri S, Powell GVN, Asner GP, Peres CA (2012) LiDAR measurements of canopy structure predict spatial distribution of a tropical mature forest primate. Remote Sensing of Environment, 127, 98-105.

Pau S, Gillespie TW, Wolkovich EM (2012) Dissecting NDVI-species richness relationships in Hawaiian dry forests. Journal of Biogeography, 39, 1678-1686.

Pausas JG, Ribeiro E (2017) Fire and plant diversity at the global scale. Global Ecology and Biogeography, 26, 889897.

Pereira HM, Ferrier S, Walters M, Geller GN, Jongman RHG, Scholes RJ, Bruford MW, Brummitt N, Butchart SHM, Cardoso AC (2013) Essential biodiversity variables. Science, 339, 277-278.

Peterson EB (2005) Estimating cover of an invasive grass (Bromus tectorum) using tobit regression and phenology derived from two dates of Landsat ETM+ data. International Journal of Remote Sensing, 12, 2491-2507.

Pettorelli N, Owen HJF, Duncan C (2016) How do we want satellite remote sensing to support biodiversity conservation globally? Methods in Ecology and Evolution, 5, 656-665.

Resasco J, Hale AN, Henry MC, Gorchov DL (2007) Detecting an invasive shrub in a deciduous forest understory using late-fall Landsat sensor imagery. International Journal of Remote Sensing, 28, 3739-3745.

Rocchini D, Balkenhol N, Carter GA, Foody GM, Gillespie TW, He KS, Kark S, Levin N, Lucas K, Luoto M (2010) Remotely sensed spectral heterogeneity as a proxy of species diversity: Recent advances and open challenges.
Ecological Informatics, 5, 318-329.

Rocchini D, Luque S, Pettorelli N, Bastin L, Doktor D, Faedi N, Feilhauer H, Feret JB, Foody GM, Gavish Y (2018) Measuring $\beta$-diversity by remote sensing: A challenge for biodiversity monitoring. Methods in Ecology and Evolution, 9, 1787-1798.

Sandino J, Wooler A, Gonzalez F (2017) Towards the automatic detection of pre-existing termite mounds through UAS and hyperspectral imagery. Sensors, 17, 2196.

Schneider FD, Morsdorf F, Schmid B, Petchey OL, Hueni A, Schimel DS, Schaepman ME (2017) Mapping functional diversity from remotely sensed morphological and physiological forest traits. Nature Communications, 8, 1441.

Schwaller MR, Southwell CJ, Emmerson LM (2013) Continental-scale mapping of Adelie penguin colonies from Landsat imagery. Remote Sensing of Environment, 139, 353-364.

Seidel D, Hoffmann N, Ehbrecht M, Juchheim J, Ammer C (2015) How neighborhood affects tree diameter incrementNew insights from terrestrial laser scanning and some methodical considerations. Forest Ecology and Management, 336, 119-128.

Sidle JG, Johnson DH, Euliss BR, Tooze M (2002) Monitoring black-tailed prairie dog colonies with high-resolution satellite imagery. Wildlife Society Bulletin, 30, 405-411.

Skidmore AK, Pettorelli N, Coops NC, Geller GN, Hansen M, Lucas R, Mücher CA, O’Connor B, Paganini M, Henrique MP, Schaepman ME, Turner W, Wang T, Wegmann M (2015) Agree on biodiversity metrics to track from space. Nature, 523, 403-405.

Skowronek S, Ewald M, Isermann M, Van De Kerchove R, Lenoir J, Aerts R, Warrie J, Hattab T, Honnay O, Schmidtlein S (2017) Mapping an invasive bryophyte species using hyperspectral remote sensing data. Biological Invasions, 19, 239-254.

Stevens N, Lehmann CER, Murphy BP, Durigan G (2017) Savanna woody encroachment is widespread across three continents. Global Change Biology, 23, 235-244.

Tang ZY, Fang JY, Sun JY, Gaston KJ (2011) Effectiveness of protected areas in maintaining plant production. PLoS ONE, 6, e19116.

Tao SL, Guo QH, Li C, Wang ZH, Fang JY (2016) Global patterns and determinants of forest canopy height. Ecology, 97, 3265-3270.

Tesfamichael SG, Newete SW, Adam E, Dubula B (2017) Field spectroradiometer and simulated multispectral bands for discriminating invasive species from morphologically similar cohabitant plants. GIScience \& Remote Sensing, 55, 417-436.

Townsend PA, Eshleman KN, Welcker C (2004) Remote sensing of gypsy moth defoliation to assess variations in stream nitrogen concentrations. Ecological Applications, 14, 504516.

Tucker CJ, Hielkema JU, Roffey J (1985) The potential of 
satellite remote sensing of ecological conditions for survey and forecasting desert-locust activity. International Journal of Remote Sensing, 6, 127-138.

Turner W (2014) Sensing biodiversity. Science, 346, 301-302.

Turner W, Rondinini C, Pettorelli N, Mora B, Leidner AK, Szantoi Z, Buchanan G, Dech S, Dwyer J, Herold M (2015) Free and open-access satellite data are key to biodiversity conservation. Biological Conservation, 182, 173-176.

Turner W, Spector S, Gardiner N, Fladeland M, Sterling E, Steininger M (2003) Remote sensing for biodiversity science and conservation. Trends in Ecology and Evolution, 18, 306-314.

Tweheyo M, Lye KA, Weladji RB (2004) Chimpanzee diet and habitat selection in the Budongo Forest Reserve, Uganda. Forest Ecology and Management, 188, 267-278.

Underwood E, Ustin S, DiPietro D (2003) Mapping nonnative plants using hyperspectral imagery. Remote Sensing of Environment, 86, 150-161.

Ustin SL, Gamon JA (2010) Remote sensing of plant functional types. New Phytologist, 186, 795-816.

Watson RM, Turner MIM (1965) A count of the large mammals of the Lake Manyara National Park: Results and discussion. African Journal of Ecology, 3, 95-98.

Wiens J, Sutter R, Anderson M, Blanchar J, Barnett A, Aguilar-Amuchastegui N, Avery C, Laine S (2009) Selecting and conserving lands for biodiversity: The role of remote sensing. Remote Sensing of Environment, 113, 1370- 1381.

Wu B, Zhu CQ, Li DQ, Dong K, Wang XL, Shi PL (2006) Setting biodiversity conservation priorities in the Forests of the Upper Yangtze Ecoregion based on ecoregion conservation methodology. Biodiversity Science, 14, 87-97. (in Chinese with English abstract) [吴波, 朱春全, 李迪强, 董珂, 王秀否, 石培礼 (2006) 长江上游森林生态区生物多样 性保护优先区确定一一基于生态区保护方法. 生物多样 性, 14, 87-97.]

Xiao Y, Ouyang ZY, Zhu CQ, Zhao JZ, He GJ, Wang XK (2004) An assessment of giant panda habitat in Minshan, Sichuan, China. Acta Ecologica Sinica, 24, 1373-1379. (in Chinese with English abstract) [肖炏, 欧阳志云, 朱春全, 赵景柱, 何国金, 王效科 (2004) 岷山地区大熊猫生境评 价与保护对策研究. 生态学报, 24, 1373-1379.]

Xin LJ, Jin YC, Zhu YP, Luo JW, Wang L, Chen B, Wang W (2015) Development of effectiveness assessment indicators of desert nature reserves in China: A case study of the Anxi National Nature Reserve. Journal of Desert Research, 35, 1693-1699. (in Chinese with English abstract) [辛利娟, 靳 勇超, 朱彦鹏, 罗建武, 王亮, 陈冰, 王伟 (2015) 中国 荒漠类自然保护区保护成效评估指标及其应用. 中国沙 漠, 35, 1693-1699.]

Xin LJ, Wang W, Jin YC, Diao ZY, Li JS (2014) Indices of ecological effects of grassland nature reserves in China. Pratacultural Science, 31, 75-82. (in Chinese with English abstract) [辛利娟, 王伟, 靳勇超, 刀兆岩, 李俊生 (2014) 全国草地类自然保护区的成效评估指标. 草业科学, 31,
75-82.]

Xu C, Holmgren M, Van Nes EH, Maestre FT, Soliveres S, Berdugo M, Kefi S, Marquet PA, Abades S, Scheffer M. (2015) Can we infer plant facilitation from remote sensing? a test across global drylands. Ecological Applications, 25, 1456-1462Yan YY, Deng J, Zhang ZQ, Zhou XY, Yang DD (2014) Research progress in the protection efficacy evaluation of wildlife nature reserves. Chinese Journal of Ecology, 33, 1128-1134. (in Chinese with English abstract) [晏玉芗, 邓娇, 张志强, 周先雁, 杨道德 (2014) 野生动物类型自 然保护区保护成效评估研究进展. 生态学杂志, 33, 11281134.]

Zellweger F, Baltensweiler A, Ginzler C, Roth T, Braunisch V, Bugmann H, Bollmann K (2016) Environmental predictors of species richness in forest landscapes: Abiotic factors versus vegetation structure. Journal of Biogeography, 43, 1080-1090.

Zhang J, Hu J, Lian J, Fan Z, Ouyang X, Ye W (2016a) Seeing the forest from drones: Testing the potential of lightweight drones as a tool for long-term forest monitoring. Biological Conservation, 198, 60-69.

Zhang J, Nielsen SE, Mao LF, Chen SB, Svenning JC (2016b) Regional and historical factors supplement current climate in shaping global forest canopy height. Journal of Ecology, 104, 469-478.

Zhang J, Rivard B, Sanchez-Azofeifa A, Castro-Esau K (2006) Intra- and inter-class spectral variability of tropical tree species at La Selva, Costa Rica: Implications for species identification using HYDICE imagery. Remote Sensing of Environment, 105, 129-141.

Zhang YJ, Fan CK, Huang K, Liu YJ, Zu JX, Zhu JT (2017) Opportunities and challenges in remote sensing applications to ecosystem ecology. Chinese Journal of Ecology, 36, 809-823. (in Chinese with English abstract) [张扬建, 范春 捆, 黄珂, 刘瑶杰, 珇佳星, 朱军涛 (2017) 遥感在生态 系统生态学上应用的机遇与挑战. 生态学杂志, 36, 809823.]

Zheng YM, Zhang HY, Niu ZG, Gong P (2012) Protection efficacy of national wetland reserves in China. Chinese Science Bulletin, 57, 207-230. (in Chinese) [郑姚闽, 张海英, 牛振国, 宫鹏 (2012) 中国国家级湿地自然保护区保护 成效初步评估. 科学通报, 57, 207-230.]

Zimmermann NE, Edwards TC, Moisen GG, Frescino TS, Blackard JA (2007) Remote sensing-based predictors improve distribution models of rare, early successional and broadleaf tree species in Utah. Journal of Applied Ecology, 44, 1057-1067.

Zou L, Miller SN, Schmidtmann ET (2006) Mosquito larval habitat mapping using remote sensing and GIS: Implications of coalbed methane development and West Nile Virus. Journal of Medical Entomology, 43, 1034-1041.

(责任编委: 李俊生 责任编辑: 时意专) 